\author{
Graham C. Holt
}

\title{
Higher education: a risk too far
}

\begin{abstract}
The protagonists of Great Britain's Industrial Revolution were, famously, typically men without a formal education. Nevertheless, the development of ultrahigh technologies such as nanotechnology seems to demand a high level of higher education. This essay appraises the appropriate level of graduate education using the concept of the J-value. Education is discussed in the context of other government services. It is concluded that higher education is an excellent national investment provided the number of graduates is maintained at an affordable level.
\end{abstract}

Nanotechnology Perceptions 8 (2012) 139-147

Nonsubscribers: purchase individual article 